\title{
Creando Geografías del Paladar en el Siglo XXI: el vino chileno y su consumo en Reino Unido
}

\author{
Robert Gwine', rngwynne@bham.ac.uk
}

\section{RESUMEN}

Este artículo intenta hacer conexiones entre los mundos de producción y los mundos de consumo, en particular introducir el concepto de "geografía de paladar" y demostrar que el concepto refleja los entendimientos de redes de comida y vino a nivel global y sus relaciones económicas y sociales. Geografía del paladar muestra cómo redes de comida y vino están vinculados a los cambios de los consumidores y cómo actores globales (compradores para supermercados) se relacionan con estos cambios en los mercados en los cuales operan. Mientras tanto, a nivel de producción de estas redes globales, la geografía del paladar demuestra la necesidad de productores de vino de cambiar sus mezclas de producción y crear nuevos gustos, debido a la percepción de estos cambios de consumo en espacios lejanos y diferentes. Se vincula el comercio internacional del vino en las zonas de producción en Chile con las zonas de consumo en su mercado principal que es el Reino Unido.

Palabras claves: Vino; producción; Chile; consumo; U.K.

\section{Creating palate geographies in the XXI century: Chilean wine and its consumption in the United Kingdom.}

\begin{abstract}
This article makes connections between the worlds of production and the worlds of consumption and introduces the concept of palate geographies. This concept reflects an understanding of food and wine networks and links them to the changes of consumers and how global actors (such as buyers from supermarket chains) relate to markets within which they operate. At the level of production, palate geographies show how the needs of wine products change their product mix and create new tastes owing to changes of consumption and markets so very different. It links the international trade of wine in zones of production in Chile with zones of consumption in its principal market, that of the United Kingdom.
\end{abstract}

Keywords: Wine; production; Chile; Consumption; U.K.

Recibido el 20 de mayo de 2015, aceptado el 5 de julio de 2015.

1 University of Birmingham, School of Geography, Earth and Environmental Sciences, Edgbaston, Birmingham B15 2TT, Reino Unido. 


\section{INTRODUCCIÓN}

El consumo del vino en el Reino Unido se ha incrementado en forma rápida y refleja un fenómeno abierto a nuevos mundos, a diferencia por ejemplo de Francia y España, que consumen vino francés y español, cuyas fuentes de consumo están vinculadas a los espacios de producción de su país. Históricamente, el consumo de alcohol en el Reino Unido ha estado más vinculado al consumo de cerveza que al vino, sin embargo, en el siglo XXI, el consumo de vino ha aumentado en forma más rápida en relación a la cerveza, en comparación con otros países Europeos continentales (como Francia), en los que el nivel de consumo per cápita ha disminuido fuertemente. Por ejemplo en Francia, el consumo de vino per cápita ha bajado de 100 litros en 1977 hasta 50 litros al comienzo del presente siglo (ROBINSON 2006).

En Francia, las importaciones de vino representan el 5\% del mercado de vino y hasta cierto punto, rechazan vinos extranjeros. Mientras tanto en el Reino Unido, hay poca producción de vino y un mínimo de restricciones para importarlo desde otros países, siempre y cuando pase los controles de calidad, que en este país son muy estrictos. El mercado británico es muy especial ya que las atracciones del Viejo Mundo (Francia, Italia y España) y del Nuevo Mundo (Las Américas, Australia, África del Sur) son muy competitivas y permiten degustar nuevos productos. La Geografía del Paladar, se ha desarrollado gracias al gran número de nuevos consumidores. En 2014, el mercado Británico importó mucho más vino del Nuevo Mundo que del Viejo Mundo.

El consumidor británico está muy consciente que los precios del producto. En 2006, el precio promedio de la botella de vino en el Reino Unido fue de $£ 3.99$ - siendo $£ 3.82$ el promedio de una botella vendida en Tesco, uno de los supermercados más grandes del Reino Unido. Actualmente el promedio de una botella de vino ha subido muy poco y es de $£ 4.25$ Los productores chilenos tienen que observar este mercado competitivo y que es bastante ventajoso para sus productos ya que hay mucha competencia entre estos mercados, pudiendo ofrecer precios más bajos. Por eso, los productores de vino chileno ven el mercado del Reino Unido como una meta muy importante entre los mercados internacionales del siglo XXI.

El concepto de Geografía del Paladar, se analiza a partir de tres temas relacionados con el productor chileno y el consumidor británico.

- El consumidor británico y el poder de los supermercados.

- Traspaso de conocimientos y cooperación entre la cadena de valor entre productores chilenos y los compradores de supermercados en el Reino Unido.

- La creación de espacios de producción de vino en Chile.

\section{CREANDO LAS GEOGRAFÍAS DEL PALADAR}

¿Es posible degustar lugares? el vino, como producto de lujo, se distingue por amplio rango en variedades. Históricamente, el consumo de vino ha estado muy vinculado a un lugar de producción cercano, como mejor ejemplo está el caso de Francia. Pero en el siglo XXI, la cantidad de lugares de producción se ha expandido considerablemente, sobre todo en el Nuevo Mundo donde hay más variedades $\mathrm{y}$ productores de vino y donde cada productor debe vincularse a nuevas redes de distribución global. La gran variedad de tipos de vino están vinculados a diferencias globales y a la posibilidad de combinarlas en forma variada. Los vinos tienen vida antes y después de aparecer en los supermercados, es decir, la Geografía del Paladar está cada vez más vinculada a distintos lugares e 
instituciones sociales y también a la manera como el vino se desarrolla desde la viña al consumidor.

La geografía relacionada con el procesamiento del vino (la tecnología manufacturera) puede proporcionar diferentes sabores o gustos para el consumidor, a partir de una misma uva. Hay una gran diferencia entre productores de vino en el valle de Rhone en Francia (Syrah) y Australia (Shiraz). La uva es la misma, pero la uva Shiraz de Australia es más dulce y más madura que la uva de Francia. Los productores en Chile han reconocido los contrastes y han desarrollado sus combinaciones entre las versiones francesas y australiana. Los consumidores ahora pueden reconocer una Geografía del Paladar de la uva Syrah/Shiraz entre productores de Francia, Australia y Chile.

En el caso de otras variedades nuevas, algunos lugares son mejores cotizados en el Nuevo Mundo que en aquellos tradicionales del Viejo Mundo. De esta manera, para la producción de Carmenere en Chile, en el siglo XXI no hay competencia entre los productores de esta variedad en el mundo, como tampoco en la región de Burdeos en Francia, donde Carmenere se comenzó a producir desde el siglo XIX.

En relación a la producción, se puede diferenciar la Geografía del Paladar por las variedades globales. La mayoría vienen de Francia (como Sauvignon Blanc y Chardonnay en blancos; Cabernet Sauvignon, Merlot, Pinot Noir y Syrah, en tintos), otras vienen de Alemania (Riesling y Gewurtztraminer), Austria (Sylvaner) e Italia (Nebbiolo). Pero a veces el origen de la uva parece más complejo, como es el caso de la Zinfandel en California. En el siglo XIX, algunos productores pensaron que esta fue una uva nativa de Estados Unidos, pero después fue analizada en forma científica y se concluyó era una uva proveniente del sur de Italia (la uva Primitiva), pero cuyo origen era de Croacia, donde se le conoce como Crljenak Kastelanski. Se puede decir entonces que la uva típica de California proviene en una cepa de orígenes complejos desde el Viejo Mundo y que la Geografía del Paladar presenta una larga trayectoria en términos de su historia.

Los países que producen vino tienen éxito si desarrollan sabores y cepas distintivas para los mercados globales, como por ejemplo, Zinfandel de California y Carmenere de Chile. Otras cepas, como Cabernet Sauvignon, del Viejo Mundo, tienen más competencia en el Nuevo Mundo debido a que hay más lugares de producción y amplían le extensión del concepto de Geografía del Paladar.

En este contexto se introduce el concepto de terroir, es decir, que la calidad especial de un producto agrícola, como un vino en particular, está determinada por las características del lugar de origen. Algunos escritores piensan que son factores relacionados con la geografía física, como los suelos, la geología, el clima y aspectos del medioambiente que crean una producción de calidad (GADE 2004). Pero otros escritores piensan que el concepto de terroir puede incluir factores humanos del pasado y cómo las habilidades y prácticas de un lugar, se traspasan de generación a generación. Un ejemplo de esto, son los pequeños productores de vino Borgoño en Francia. Sin embargo MORAN (2001) citó a Louis Latour, un comerciante de Borgoño que dijo que cuando una persona analiza los elementos de terroir, existen tantas incertidumbres que es peor identificar y analizar los elementos que lo conforman. Sin embargo, dijo que el terroir es una herramienta para comercializar un producto en forma excelente.

De todas maneras hay una distinción crítica entre el concepto de terroir del Viejo Mundo y la del Nuevo Mundo. En el Viejo Mundo, el concepto mira hacia el pasado; la identificación de calidad de producción mira a sus raíces rurales e intenta combinar 
los elementos de la geografía, tanto físicos como humanos. Mientras tanto en el Nuevo Mundo, se está construyendo y desarrollando el concepto de terroir; Las empresas de vino miran su presente y su futuro, para crear la identidad de la producción de vino. El concepto de terroir es una combinación de factores geográfico físicos y de aplicaciones tecnológicas modernas. Los factores genéticos y del medio ambiente son importantes para el consumidor en su selección de un tipo del producto.

El concepto de terroir en Chile y en el Nuevo Mundo ha sido creado en el contexto de un sistema de mercado. Las decisiones de las empresas, tanto individuales como colectivas, en términos de una identidad de un lugar, son importantes para crear el reconocimiento global de un producto proveniente de un determinado lugar. Para demostrar al público que un vino posee asociado el concepto de terroir es a través de la información al consumidor respecto de las características especiales y únicas características que posee.

El concepto de terroir en el Nuevo Mundo permite hacer conexiones entre productores y consumidores; en California, está vinculado a las prácticas sociales, políticas y económicas. Las empresas productoras de vino tratan de hacer conexiones entre la calidad y el lugar de producción. Este vínculo puede conectar la naturaleza (clima, suelos y paisaje) y los rasgos del lugar. El valle de Colchagua en Chile, es un calro ejemplo de esta situación.

\section{LA GEOGRAFÍA DEL PALADAR, LAS CADENAS DE VALORES Y LOS SUPERMERCADOS GLOBALES}

En las regiones de los países del Nuevo Mundo, se puede discutir que en la indicación geográfica, la empresa tiene que crear el concepto, sobre todo las empresas del vino y de las organizaciones vinculadas a esta. Esto introduce el concepto de mercados calificados, vale decir, mercados en los cuales la definición de calidad está directamente relacionada con la interacción entre productores y consumidores. Se puede discutir que la calidad es una cuestión de competencia o de cooperación entre actores en la cadena de valor que incorpora distintos actores como por ejemplo, en el país de producción (el productor de uvas-la bodega de producción-empresas de exportación) y en el país de consumo (empresas de importación-distribuidores-supermercadosconsumidores). Cada actor tiene acceso a la información de esta cadena de valor. Los productores tienen acceso a la información del producto: la producción de los vinos y el procesamiento manufacturero, sin embargo, no tienen la información de cómo el producto influencía al consumidor en los mercados globales, ya que los distribuidores y supermercados tienen un mayor control y más información. Se hace necesario que la calidad en la cadena de valor esté asociada a cooperación y conocimiento existente entre los diferentes actores beneficiándose así todos los miembros de la cadena de valor.

La Geografía del Paladar, puede concentrarse en el vínculo existente entre aspectos de producción (físico, cultural y económico) y aspectos de consumo (cultural, económico y geográfico). Así entonces se puede explicar el análisis del gusto de un producto y los orígenes en el territorio: ¿Quiénes son los actores? El consumo está cada vez más organizado por un pequeño número de empresas grandes que son los proveedores.

Algunos autores discuten que la cadena de valor está más controlada por los consumidores y que los supermercados son los actores principales. Aunque los supermercados no tienen acceso directo 
a la tecnología de los productores, estos pueden exigir requisitos, en este caso a las empresas de producción y de exportación en países de producción. Los productores de vino necesitan la nueva tecnología de otras fuentes y con los enólogos internacionales que viajan hacia los países del Viejo y Nuevo Mundo, se logra este conocimiento, que facilita los requisitos de un supermercado donde los compradores piden degustar vinos nuevos que han sido desarrollados en ambos mundos y que han usado combinaciones de diferentes uvas.

\section{EL CONSUMIDOR Y EL SUPERMERCADO: RIESGOS Y ESFUERZOS}

Las estrategias de los supermercados han permitido desarrollar la imagen de una marca para aumentar la importancia de los productos propios, hasta un 50\% durante el siglo XXI. Esto significa que los supermercados han generado un papel activo en la innovación de productos y dirección de abastecimiento. En términos del consumidor en el Reino Unido, se puede adaptar un modelo de jerarquía en relación al riesgo relativo y esfuerzos de compra (Fig.1).

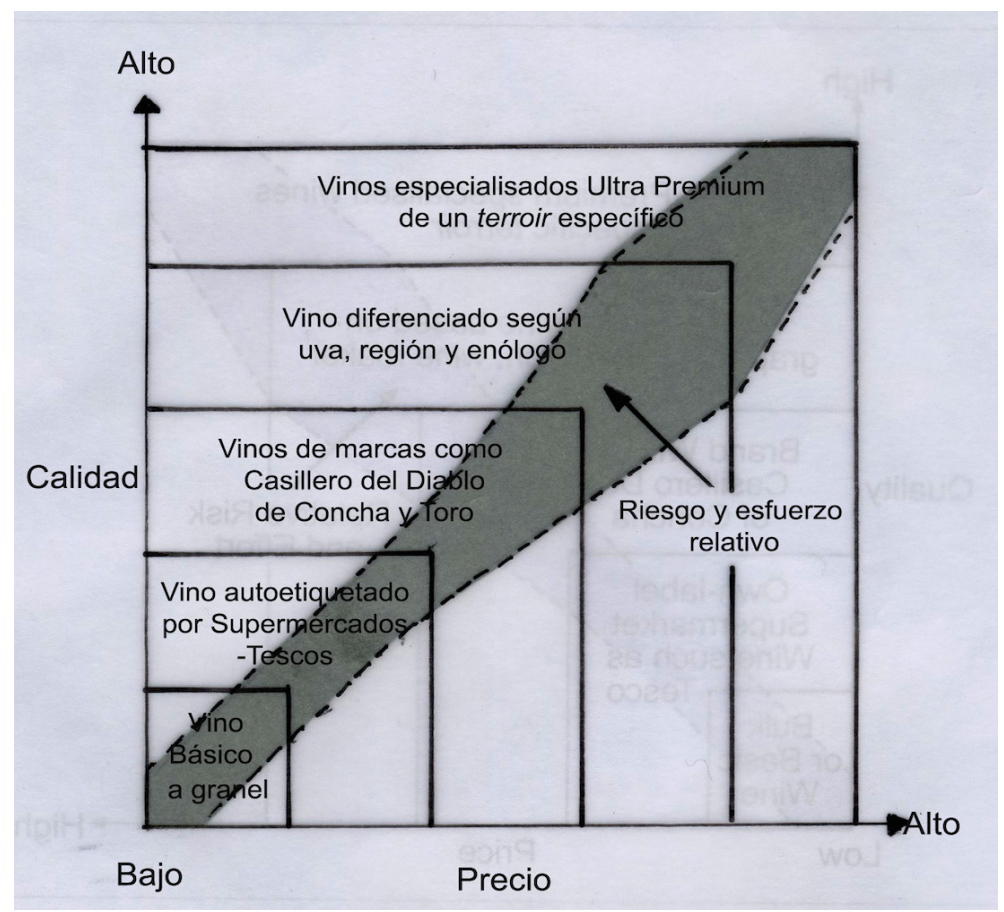

Fig. 1. Jerarquía basadas en precio, calidad, esfuerzo y riesgo para el consumidor del vino Chileno en el mercado Británico (adaptado de SPAWTON 1991)

Fig. 1. Hierarchical ranking and the price, quality, effort and risk classification for consumers of Chilean wine products in the UK Market (adapted from SPAWTON 1991) 
Se puede ver que el riesgo y los esfuerzos de compra para el consumidor británico están relacionados en términos de comprar vino barato o marcas producidas por el supermercado. En la Figura 1, los ejes de calidad de precio y las clasificaciones del producto de vino, no son representativas de las proporciones del mercado. Pero en el siglo XXI, muestra que uno de los supermercados más grandes en el Reino Unido, Tesco, tiene alrededor del 50\% de sus ventas de vino en sus marcas propias. Los supermercados han definido estas estrategias usando marcas etiquetadas por la compañía y estos han tenido éxito en mostrar marcas etiquetadas como de calidad y no como una marca barata.

Los supermercados reflejan los estilos de vida de sus consumidores. En el Reino Unido, el consumo de vino per cápita está aumentando y hay más interés en desarrollar sus propias etiquetas para atraer a los consumidores que toman vino como parte de su estilo de vida e inculcarles que pueden comprar un vino de mejor calidad, pero más barato. Los analistas se enfocan en "el momento de tomar su vino" y encuentran que hay cuatro temas importantes: sentimientos de escapismo e indulgencia; conexiones con la familia, los amados y los amigos; reducción del estrés y relajación; sofisticación (HALSTEAD 2006). Al momento de tomar su vino es cuando el consumidor recibe el impacto del gusto que el enólogo quiso impartir con la combinación final de vinos, que seleccionó hace meses o años atrás para cumplir los requisitos del supermercado. Tomar vino puede ser una ocasión netamente personal o netamente sociable $y$ hay muchas diferencias de género entre estas ocasiones.

La discusión de la Geografía del Paladar puede reflejar el conocimiento del consumidor para decidir una región favorita o una cepa global. En el Reino Unido, las mujeres seleccionan la cepa Chardonnay como su vino blanco, sin embargo, el conocimiento para elegir regiones de vino no es tan importante.

La Geografía del Paladar permite la interacción del consumidor con regiones que producen vino. Con conocimientos, la decisión de elegir una región de vino o el proceso de producción, puede ser importante, como por ejemplo: vino que es fair trade u orgánico y puede llevar a la reflexión y asociación entre lugares, la calidad del vino o la política del comprador. ¿De qué manera puede él/ella consumidor/a del Reino Unido elegir lugares de vino en Chile?

\section{LOS CONSUMIDORES BRITÁNICOS Y LA VIÑA CHILENA AL COMIENZO DEL SIGLO XXI}

El consumidor británico puede elegir vino de distintas regiones en Chile. Según la viña, Luis Felipe Edwards, los consumidores británicos están "cinco años al frente del consumidor en el resto del mundo en términos de gusto, precio y requisitos". Ello significa que el consumidor británico está abierto a nuevos gustos y lugares de producción del vino de Chile.

Al comienzo del siglo XXI, dos organizaciones de vino Chileno (Asociación de Viñas de Chile y Chilevid) reconocieron las estrategias del mercado del vino en el Reino Unido y crearon "Wines of Chile" en 2002, como una organización sin fine de lucro. La estrategia fue construir una oficina fuera de Chile y aumentar las ventas de vino en el mercado del Reino Unido; también lo fue mostrar un modelo nuevo de negocios en otros mercados del mundo. "Wines of Chile" comenzó en Junio del 2003 y registró un éxito significativo al aumentar la venta de vino Chileno en el Reino Unido. La proporción de vino Chileno aumentó de $5.9 \%$ en 2003 hasta $6.9 \%$ en 2007 y $8 \%$ en la venta de restaurantes y bares. En el 2007, Chile tuvo la proporción más alta 
en el Reino Unido, después de Australia y Estados Unidos, entre los países del Nuevo Mundo.

Los supermercados en el Reino Unido piensan que la venta de vino puede atraer mejores consumidores en términos de cantidad de ventas, pues tienen flexibilidad para desarrollar más contratos con productores de vino en el Nuevo Mundo y aumentar la extensión y calidad de sus vinos. En la figura 1 se puede ver la relación entre los supermercados y los consumidores. El concepto está basado en el riesgo y en el esfuerzo para atraer los consumidores; el papel de las marcas auto-etiquetadas de vino Chileno es un factor importante. En términos de uno de los supermercados más grandes en el Reino Unido, su vino Chileno, auto-etiquetado logra casi el 50\% de las ventas en todas sus sucursales. La confianza de los consumidores en la calidad del producto, da suficiente libertad al supermercado para introducir vino chileno y reducir la confusión del cliente. De los 22 vinos tintos en supermercado (Tesco) entre 2006 y 2010, solamente siete tintos fueron auto-etiquetados. Cuando el consumidor británico compra un vino auto-etiquetado, la compra necesita menos riesgo y esfuerzo. Los consumidores creen que la compra de un producto chileno auto-etiquetado por ese supermercado ofrece mejor calidad que la imagen de una etiqueta de otro vino.

Después de la compra de marcas autoetiquetadas, el riesgo y esfuerzo de los consumidores está vinculado solo con una marca chilena: Casillero del Diablo. En relación a mayor riesgo y esfuerzo, la calidad del vino aumenta de acuerdo a que sean vinos de cepas más diferenciadas regionalmente y de los enólogos. Un tanto más complicado y con mayor riesgo y esfuerzo para el consumidoir son los vinos muy especializados de algún terroir distintivo, como Clos Apalta de Casa Lapostolle. El mercado de vino chileno en el Reino Unido tiene dos elementos importantes: la venta en restaurantes y bares
(21\%) y la venta de supermercados grandes y cadenas de vino $(79 \%)$.

¿Qué piensa el consumidor británico de Chile y del vino chileno? Una de las respuestas es que el vino Chileno se compara con el vino del Viejo Mundo, como el vino francés. En el mercado británico, la marca chilena está asociada con su valor y el precio de venta. La mayoría de los consumidores tienen poco conocimiento de Chile y pocos viajan a este país, en comparación con sus viajes a España y Francia donde el consumidor puede degustar vinos locales. Por eso las decisiones de los supermercados son fundamentales para que el consumidor pueda elegir vino chileno.

\section{CONCLUSIONES BASADAS EN EL CASO DE COLCHAGUA, CHILE}

La Geografía del Paladar es fundamental para vincular a los consumidores británicos con los productores de vino en Chile. Los supermercados constituyen una conexión importante entre el consumidor y el productor, de manera que demandar el producto en términos de los requisitos del consumidor. El valor de la cadena global es un proceso que ha favorecido este vínculo al comienzo del siglo XXI.

¿De qué manera las regiones de Chile han producido vinos que se reconocen en el Reino Unido?. Al examinar la legislación chilena de 1995 se puede establecer que ésta permitió una distribución de la producción de vino más transparente, ya que a) si una uva de variedad fue clasificada en la etiqueta, se tuvo que abastecer por lo menos del $85 \%$ de la uva de la región; b) si una región fue mencionada en la etiqueta, el $85 \%$ de la producción tuvo que ser abastecida por productores de uva en ésta.

El rol de escritores de reconocimiento global, como Robert Parker en Estados Unidos o Jancis Robinson en el Reino Unido, han sido fundamentales en la clasificación de 
las regiones vitivinícolas. Estos escritores han influido en los consumidores para que estos decidan respecto a las regiones de producción de vinos en Chile.

Los supermercados fueron fundamentales para que los consumidores británicos reconocieran el valle de Colchagua. Los vinos tintos tienen mucha fama en el Reino Unido, como las cepas Cabernet Sauvignon, Syrah y Carmenere. En los años de 1990, inversiones significativas de Casa Lapostolle, Montes Montgras y otras empresas comenzaron a cambiar la calidad de vino del valle. Después de 1995, el valle de Colchagua fue más conocido por sus niveles de calidad de producción. Casa Lapostolle y Montes son reconocidas como productores de alto nivel en Chile y el Reino Unido. En 2006, el valle de Colchagua fue adjudicado con el premio de Global Wine Valley of the Year por una revista norteamericana, Wine Enthusiast; fue la primera región vitivinícola clasificada de este modo en Chile. La indicación geográfica del Valle del Colchagua tiene reconocimiento global.

Los productores chilenos han desarrollado un nuevo sabor a partir de una cepa que solo produce en Chile, el "resucitado" Carmenere, que fue originaria de Burdeos. Después de la enfermedad de la filoxera (Phylloxera vastatrix) en Burdeos durante los años 1870, los productores franceses no plantaron nuevamente estas uvas y finalmente se extinguió la cepa; la uva ya había sido llevada a Chile. Durante los años 1990 cuando Chile comenzó a exportar vinos se reconoce la cepa. Después de 1994 la producción de Carmenere se fomentó en muchas regiones de Chile, y en el valle de Colchagua en particular. La uva Carmenere en Chile se reproduce con vigor, madura muy lento y se cosecha en mayo (otoño). En la región se han producido variedades nuevas de vinos que antes no fueron reconocidas, generando así una Geografía de Paladar excepcional.
Con este artículo se demuestra cómo se generan espacios de producción en el Nuevo Mundo, es este caso Chile, con lugares de consumo como el Reino Unido y también el contexto global. Expone cómo la región de Colchagua ha cambiado su producción a nivel global creando una industria y una agricultura de exportación para regiones de consumo muy lejanas.

\section{REFERENCIAS}

BARHAM, E., 2003. Translating Terroir: The Global Challenge of French AOC Labelling Journal of Rural Studies, 19, 1, 127-138

BISSON, L. F., A. L. WATERHOUSE, S. E. EBELER, M. A. WALKER \& J. T. LAPSLEY, 2002. The Present and Future of the International Wine Industry, Nature, 418, 696-99.

COOK, I., 2004. Follow the Thing: Papaya, Antipode, 36, 4, 642-64

COOK, I., P. CRANG \& M. THORPE, 1998. Biographies and Geographies: Consumer Understandings of the Origins of Foods, British Food Journal, 100(3): 162-167.

DUIJKER, H., 1999. The Wines of Chile. Utrecht, Spectrum.

FANET, J., 2004. Great Wine Terroirs. Berkeley, University of California Press.

FRIEDLAND, W. H., 2005. Commodity Systems: Forward to Comparative Analysis. In: FOLD, N \&

B. PRITCHARD (eds) Cross-continental Agro-food Chains, 25-38. London, Taylor $\&$ Francis. 
GADE, D. W., 2004. Tradition, Territory and Terroir in French Viticulture: Cassis, France and Appelation Controlee. Annals of the Association of American Geographers, 94(4): 848-67.

GEREFFI, G., 1994. The Organization of Buyer-driven Global Commodity Chains: How US Retailers Shape Overseas Production Networks. In GEREFFI, G \& M. KORZENIEWICZ (eds) Commodity Chains and Global Capitalism, 67-92. Westport, Praeger.

GEREFFI, G., 1999. International Trade and Industrial Upgrading in the Apparel Commodity Chain, Journal of International Economics, 48, 37-76.

GIBBON, P. \& S. PONTE, 2005. Trading Down: Africa, Value Chains and the Global Economy. Philadelphia, Temple University Press.

GUTHEY, G., 2004. Wine, Terroir and Agro-industry in Napa and Sonoma Counties. Annual Conference of the Association of American Geographers, Philadelphia, March 17

GWYNNE, R. N., 2003. Transnational Capitalism and Local Transformation in Chile, Tijdschrift voor Economische en Sociale Geografie, 94, 310-21.

HALSTEAD, R., 2006. Understanding the Wine Drinking Moment. In: XXIX World Congress of Wine and Vine, Logrono, Spain.

HAYWARD, D. \& N. LEWIS, 2008. Regional Dynamics in the Globalizing Wine Industry: The Case of Marlborough, New Zealand, The Geographical Journal, 2:124-137.

HUMPHREY, J., 2006. Policy Implications of Trends in Agribusiness Value Chains, The European Journal of Development Research, 18(4):572-592.
HUMPHREY, J. \& H. SCHMITZ, 2002. How Does Insertion in Global Value Chains Affect Upgrading in Industrial Clusters?, Regional Studies, 36(9): 1017-27.

INTERNATIONAL WINE AND SPIRIT RECORD (IWSR), 2007. The UK Wine Market Turns into a Share Game, Drinks Record.

LAGENDIJK, A., 2004. Global "Lifeworlds" Versus Local "Systemworlds": How Flying Winemakers produce Global Wines in Interconnected Locales, Tijdschrift voor Economische en Sociale Geografie, 95(5):511-526.

MORAN, W., 1993. The Wine Appelation as Territory in France and California, Annals of the Association of American Geographers, 83(4): 694-717.

RICHARDS, P., 2006. The Wines of Chile. London, Mitchell Beazley.

ROBINSON, J., 2006. The Oxford Companion to Wine, Oxford, Oxford University Press (ed).

SANCHEZ HERNANDEZ, J. L., 2004. La Liberalizacion Controlada del Mercado del vino en Noruega 1996-2002, Investigaciones Geograficas, 35: 85-102.

SCHMITZ, H. \& P. KNORRINGA, 2000. Learning from Global Buyers, Journal of Development Studies, 37(2): 177-205.

SPAWTON, T., 1991. Wine and the Marketing Mix, European Journal of Marketing, 5(3): 19-32.

STEVENSON, T., 2005. The Sotheby's Wine Encyclopedia, London, Dorling Kindersley. 


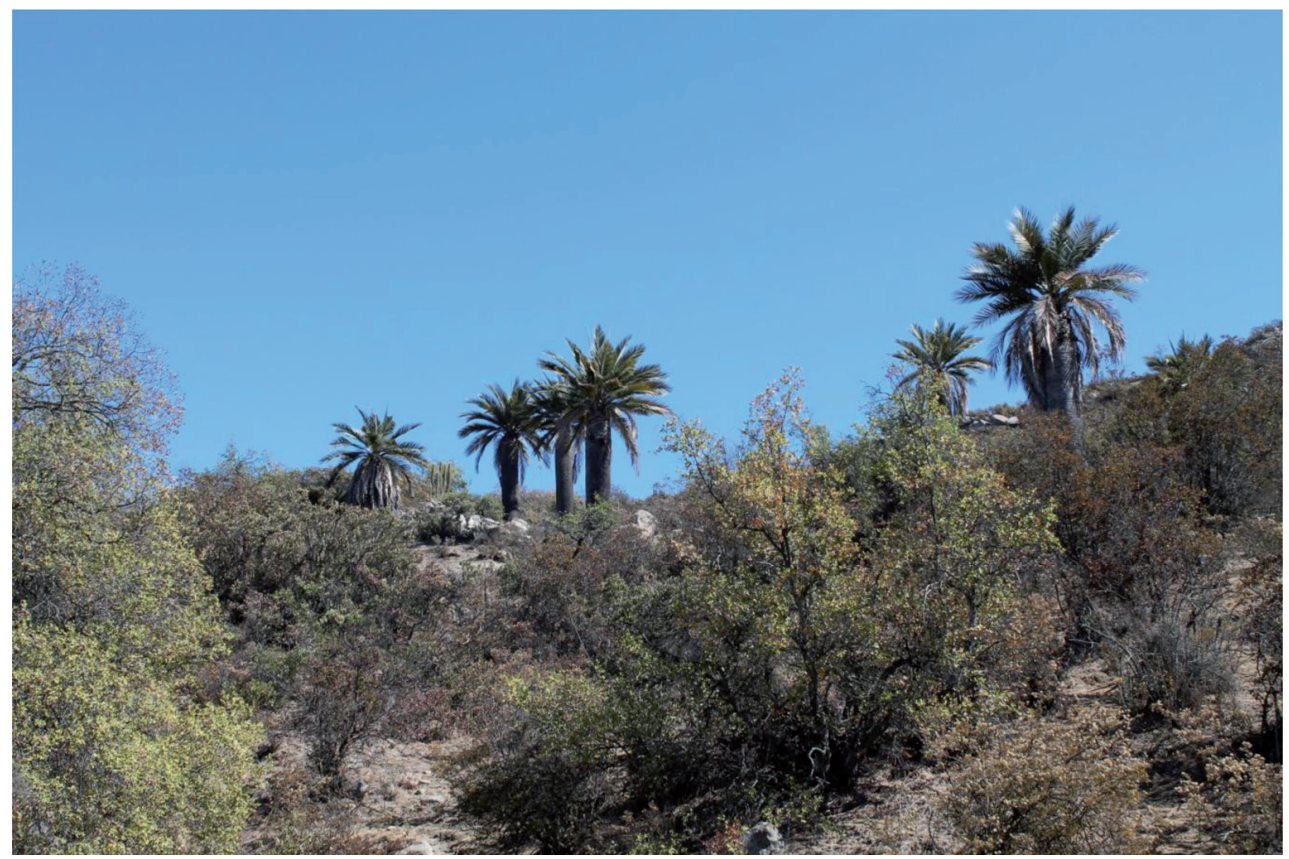

Palmas of de Tilama. Groups of long-lived Chilean palm (Jubaea chilensis (Moll) Baillon) in semiard landscape of Region de Coquimbo, Tilama zone, province of Choapa. MECESUP Project, Nº91512QP-2015, Prof. Víctor Quintanilla Pérez, victor.Quintanilla@usach.cl 